\title{
Delicate Pancreatic Rotating Technique Using "Thoraco Cotton" in Laparoscopic Gastrectomy
}

\author{
Masanari Shimada ${ }^{*}$, Susumu Amaya, Keisuke Tatemichi, Hiroki Tawara, Yuichirou Furutani, Hiroaki Sugita, Teruo \\ Okude, Hayato Suzuki, Yoshinori Munemoto and Takeshi Mitsui
}

Department of surgery, Fukui Saiseikai Hospital, Fukui, 918-8503, Japan

\section{Abstract}

Background: The securement of a field of view by pancreatic rotating technique for suprapancreatic lymphnode dissection in laparoscopic gastrectomy (LG) has a huge effect on the accuracy of dissection and is associated with the frequency of pancreatic fistula. Here, we report a delicate, safe and easy rotation technique of pancreas with "Naruke Type Thoraco Cotton" (TC; KENZMEDICO).

Methods: We evaluated the data of 34 patients who underwent laparoscopic distal gastrectomy (LDG) with suprapancreatic lymphadenectomy for gastric cancer. TC was used to compress the pancreas to secure the field of view for lymphadenectomy. During we underwent the suprapancreatic lymphnode dissection, the assistant inserted the TC from $12 \mathrm{~mm}$ trocar with the left hand, and applied pressure to the lower surface of pancreas. We showed the clinicopathologic characteristics, operative outcomes, intraoperative complications, serum amylase concentration (s-AMY) on postoperative day 3 (POD3), and amylase concentration of drainage fluid (d-AMY) on POD3, and rates of postoperative pancreasrelated complications.

Results: The procedures of lymphadenectomy were undertaken smoothly and clearly with TC. The number of the cases of postoperative pancreas-related complication was only one with D2 lymphnode dissection (Grade2 pancreatic fistula; 2.9\%). The median values of d-AMY on POD3 were only 179 IU/1. Conclusions: TC should be considered as a useful option in the safe and precise pancreatic rotation technique for suprapancreatic lymphnode dissection.

\section{Introduction}

The securement of a field of view by pancreatic rotating technique for suprapancreatic lymphnode dissection in laparoscopic gastrectomy (LG) has a huge effect on the accuracy of dissection and is associated with the frequency of pancreatic fistula. Currently there are some pancreatic rotating techniques with forceps, gauze, sponge etc. [1-3], however these methods often are not able to conduct consistent performances because they are dependent on the skills and experiences of surgical assistants. We previously reported an easy and safe pancreatic rotating technique with a balloon-shaped retractor (Cat Hand; HAKKO CO., LTD, Nagano, Japan)[4]. Here, we report a more delicate method with "Naruke Type Thoraco Cotton (TC; KENZMEDICO, Saitama, Japan).

\section{Material and Methods}

\section{Patients}

We reviewed 34 patients with gastric cancer who underwent laparoscopic distal gastrectomy (LDG) with suprapancreatic lymphadenectomy using TC between April 2014 and October 2015 at our hospital. The indications for laparoscopic gastrectomy included the following: clinical diagnosis of the depth of tumor invasion limited to the subserosa (T1 or T2, according to the Japanese Classification of Gastric Carcinoma [5]), absence of significant lymph node swelling and distant metastases in preoperative examinations, and neoplasm of any histologic type.

\section{Introduction of the surgical instrument}

TC is a simple implement composed of cotton stick and external cylinder, which was originally created by Dr. Tsuguo Naruke (ex.

\section{Publication History:}

Received: October 16, 2017

Accepted: December 09, 2017

Published: December 11, 2017

Keywords:

Laparoscopic gastrectomy, Suprapancreatic lymphadenectomy, Thoraco Cotton, Pancreatic fistula assistant director of National Cancer Center, Japan, 1934-2006) for thoracic surgery. Product catalog show an example of using TC in thoracic surgery. TC is useful for dissection, compression, hemostasis, rotation, and lifting up in various operations (Figure 1). We put TC into practice in suprapancreatic lymphadenectomy during laparoscopic gastrectomy for potential less-invasive effects.

\section{Patients}

Figure 2-6 shows the surgical process for suprapancreatic lymphadenectomy with TC. For the example, we show a 56-yearold female case. She was diagnosed with poorly differentiated adenocarcinoma (M, Gre, 0-IIc, 20mm, 0-IIc, T2, N0, M0; clinical stage IB in Japanese Classification of Gastric Carcinoma), so we performed laparoscopic distal gastrectomy with D2 lymphnode dissection.

At first, a mini laparotomy was performed in umbilical region and a $12 \mathrm{~mm}$ trocar for laparoscope was inserted. After $12 \mathrm{~mm}$ trocars were set up in the bilateral lateroabdominal region and $5 \mathrm{~mm}$ trocars were set up in the bilateral hypochondriac region, we started the laparoscopic surgery.

"Corresponding Author: Dr. Masanari Shimada, Department of surgery, Fukui Saiseikai Hospital, 7-1 Wadanaka-Funabashi, Fukui 918-8503, Japan, Tel: +81776-23-1111; E-mail: masanari.shimada@gmail.com

Citation: Shimada M, Amaya S, Tatemichi K, Tawara H, Furutani $\mathrm{Y}$, et al. (2017) Delicate Pancreatic Rotating Technique Using "Thoraco Cotton" in Laparoscopic Gastrectomy. Int J Gastroenterol Disord Ther 4: 133. doi: https:// doi.org/10.15344/2393-8498/2017/133

Copyright: (c) 2017 Shimada et al. This is an open-access article distributed under the terms of the Creative Commons Attribution License, which permits unrestricted use, distribution, and reproduction in any medium, provided the original author and source are credited. 
Citation: Shimada M, Amaya S, Tatemichi K, Tawara H, Furutani Y, et al. (2017) Delicate Pancreatic Rotating Technique Using "Thoraco Cotton" in Laparoscopic Gastrectomy. Int J Gastroenterol Disord Ther 4: 133. doi: https://doi.org/10.15344/2393-8498/2017/133

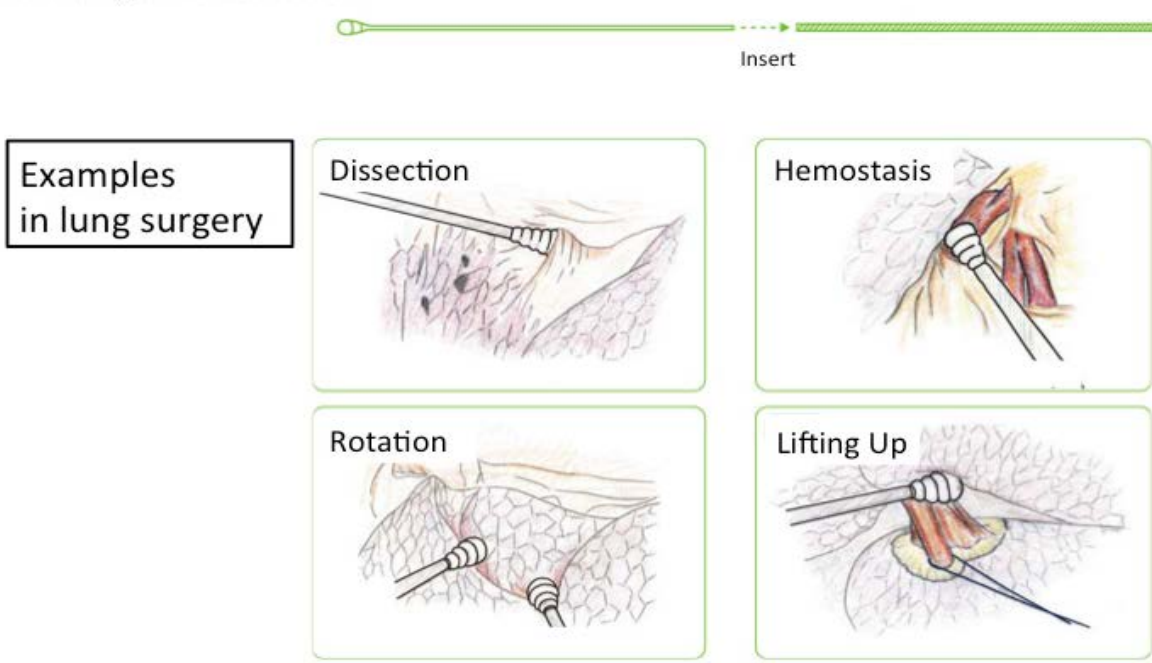

Figure 1: Examples of how TC is used.

Examples of using TC in thoracic surgery are shown in the product catalogue. TC has various functions as dissection, compression, hemostasis, rotation, and lifting up.

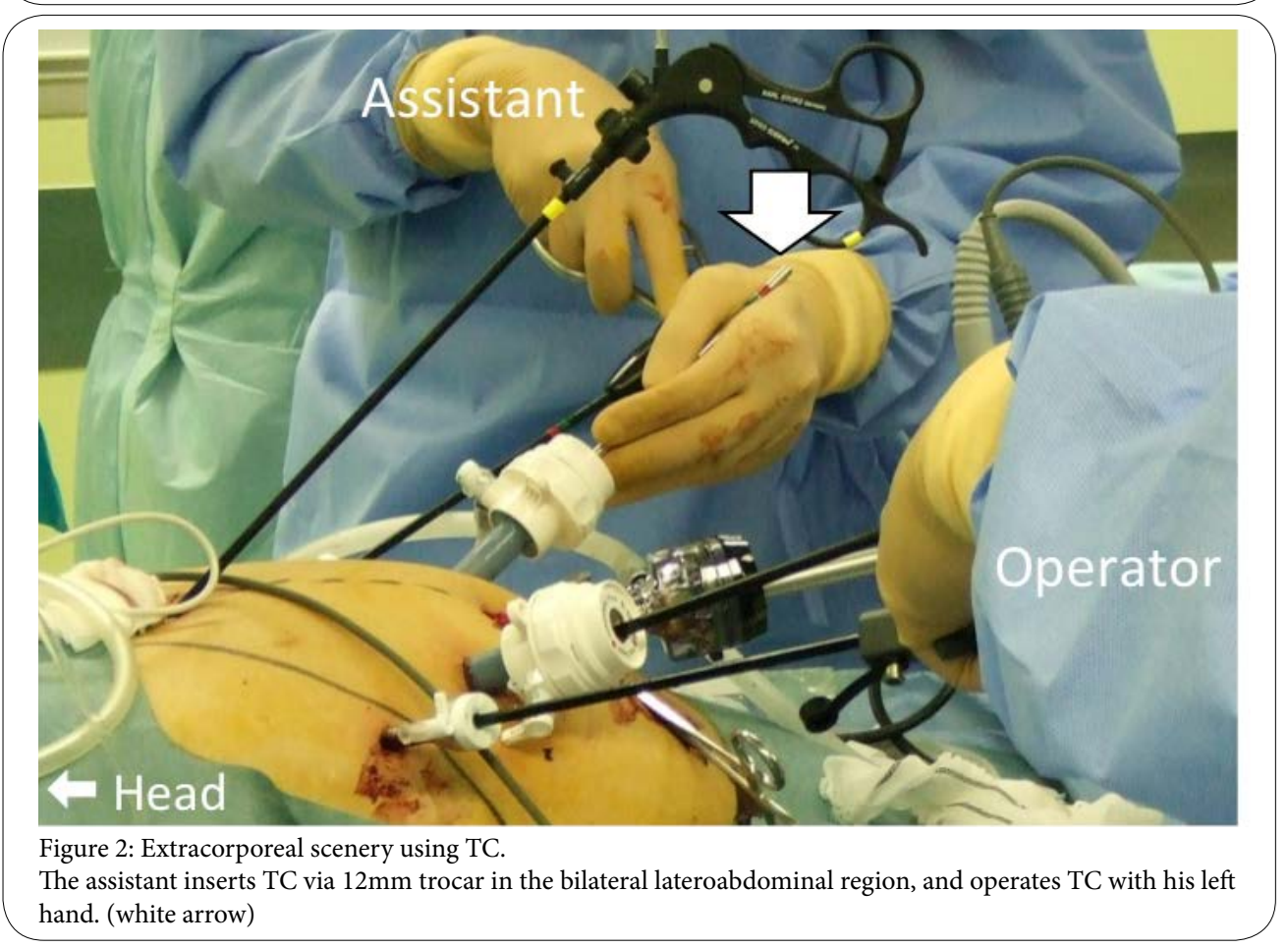

We start LG as usual and cut the duodenum before suprapancreatic lymphadenectomy, the assistant inserts the TC via $12 \mathrm{~mm}$ trocar in the bilateral lateroabdominal region (Figure 2). The assistant compresses the lower edge or the surface of the pancreas softly with TC and rotate it dorsocaudally (Figure 3). Subsequently, we can fine-tune the TC position according to the location of lymphnodes and vessels which we are going to dissect (Figure 4-6). The TC can also be used for hemostasis by compression when we have a slight hemorrhage. We remove the TC from the trocar and exchange it for other forceps rapidly after suprapancreatic lymphadenectomy is finished.

\section{Data collection and measurements}

Gender, age, BMI, surgical stages of gastric cancers, extent of lymphadenectomy, operation time, estimated blood loss, and intraoperative complications were recorded retrospectively. Amylase concentration of blood serum (s-AMY) and drainage fluid (d-AMY) were measured on postoperative days (POD) 3 to evaluate the presence of postoperative pancreas-related complications. 
Citation: Shimada M, Amaya S, Tatemichi K, Tawara H, Furutani Y, et al. (2017) Delicate Pancreatic Rotating Technique Using "Thoraco Cotton" in Laparoscopic Gastrectomy. Int J Gastroenterol Disord Ther 4: 133. doi: https://doi.org/10.15344/2393-8498/2017/133

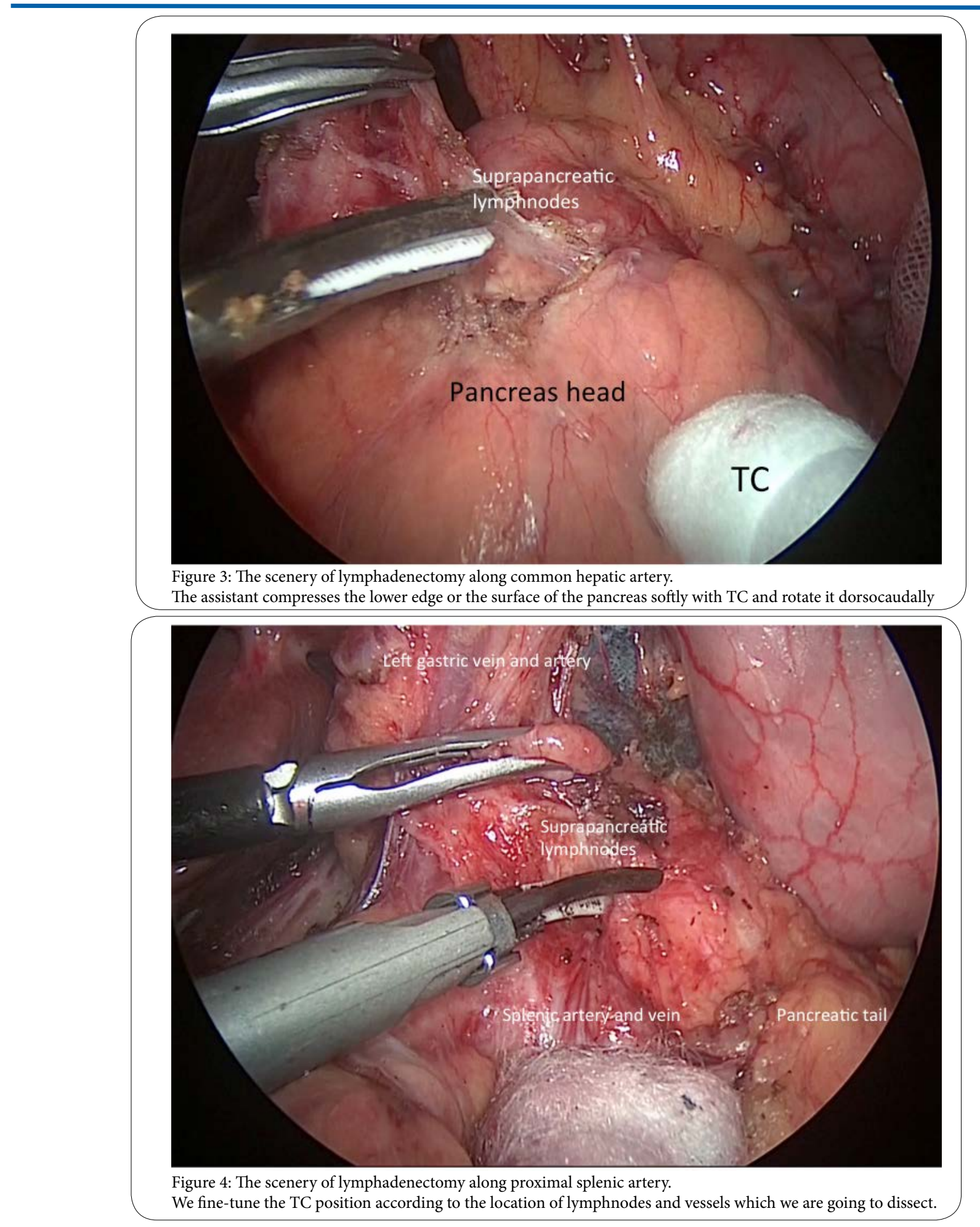

\section{Definitions of surgical complications}

We employed the International Study Group on Pancreatic Fistula Definition (ISGPF). Postoperative pancreatic fistula (POPF) is defined as a drain output of any measurable volume of fluid on or after POD 3 with d-AMY greater than 3 times the serum amylase activity [6]. The severity of POPF and other postoperative pancreas-related complications were graded by the Clavien-Dindo classification for surgical complications [7].

\section{Results}

Details of the clinical features and the perioperative measurements of LDG cases with TC are shown in Table 1 . The mean patient body mass index was $21.5 \mathrm{~kg} / \mathrm{m}^{2}$. Regarding the surgical stage and the extent of lymphadenectomy, 3 patients were changed their stage to IIB or IIIA because some lymphnode metastases were suspected in intraoperative findings. For such patients diagnosed with advanced cancer, D2 lymphadenectomy was performed in 5 patients (14.7\%). 
Citation: Shimada M, Amaya S, Tatemichi K, Tawara H, Furutani Y, et al. (2017) Delicate Pancreatic Rotating Technique Using "Thoraco Cotton" in Laparoscopic Gastrectomy. Int J Gastroenterol Disord Ther 4: 133. doi: https://doi.org/10.15344/2393-8498/2017/133

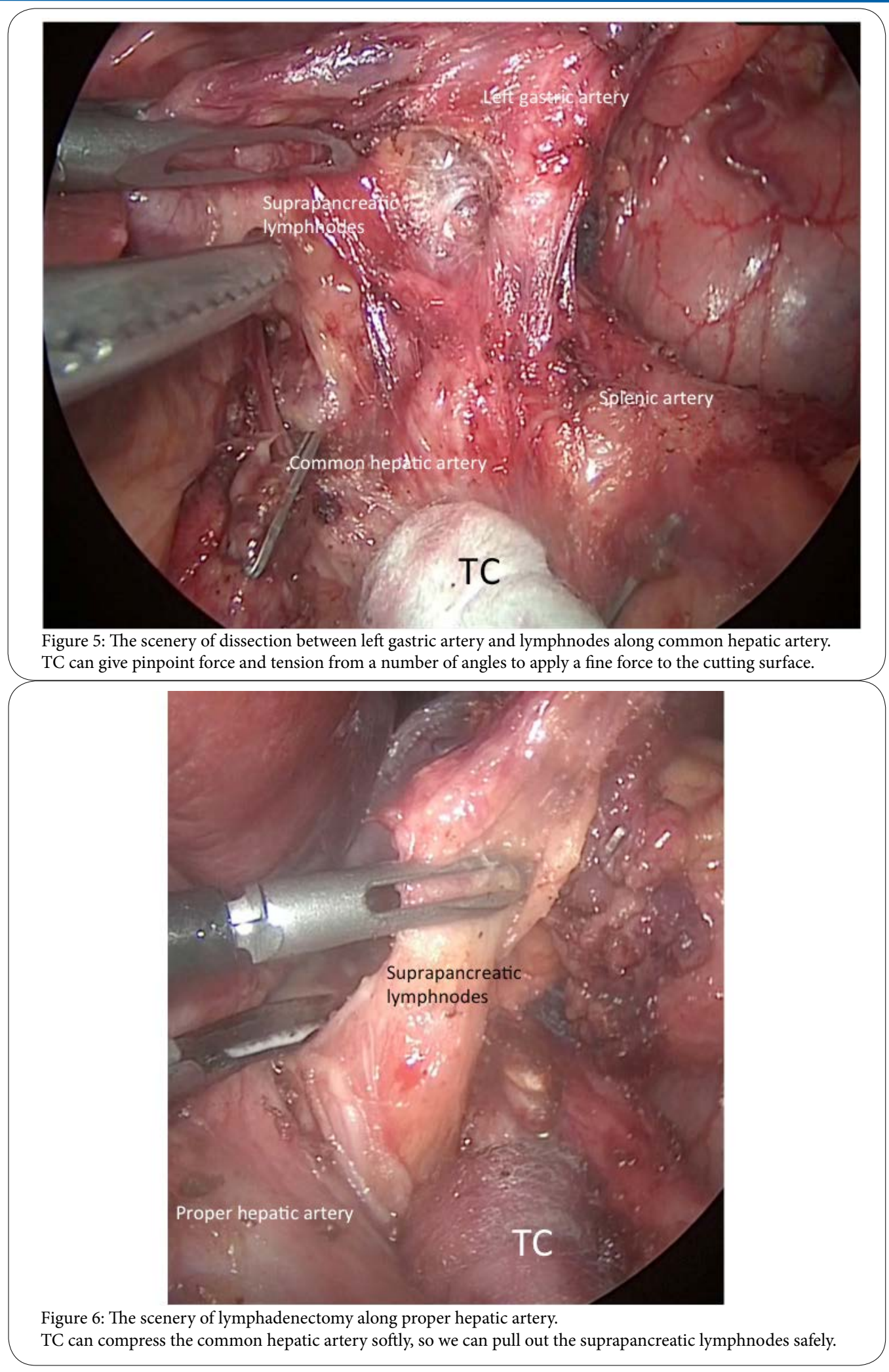

Mean operation time was 229 minutes, and mean blood loss was 22 g. There were no intraoperative complications and no conversions to open surgery in all cases. POPF grade 2 was observed in only 1 case of surgical stage IIIA (2.9\%). Other postoperative pancreas-related complication such as pancreatitis or peripancreatic abscess were not observed.

\section{Discussion}

LG has become as popular in the past several years, because it was published on various guidelines in certain limited conditions $[8,9]$. However, the distinctive procedure of compressing the pancreas dorsally is extremely important to complete safe and thorough 


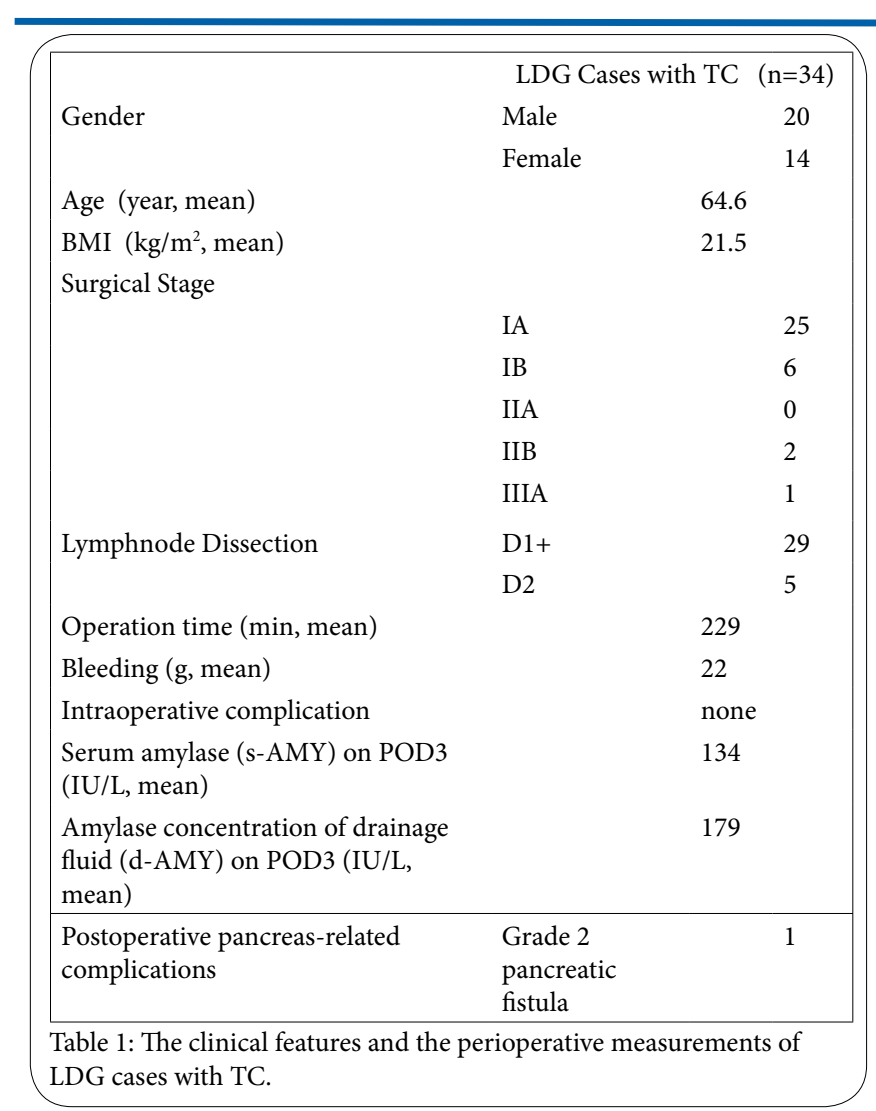

suprapancreatic lymphadenectomy in LG. Indeed, in the method with gauze, the misalignment and resetting of the gauze often breaks continuity of the surgery, and surgeons are getting stressed. On the other hand, there are some concerns about injury of the pancreatic capsule due to direct contact with the hard tip of the forceps. We have considered that such compression and rotation technique influences refinement or accuracy of the surgery, and previously reported the easy and safe rotation technique of pancreas with Cat Hand ${ }^{\circ}$ [4]. There are few literatures which describe new methods about the compression or rotation technique, consequently we report a method using TC in order to improve the accuracy of suprapancreatic lymphadenectomy.

TC's advantages which we suggest are:

1. No action for shifting the gauzes etc. is necessary.

2. There is minimal risk to organ injury.

3. The soft cotton can stop the some minute bleeding quickly by compression.

4. Delicate and pinpoint tension can be applied to the dissection site.

5. Thereby relieving stress of the surgeon / assistant.

6. The cost is very inexpensive for $¥ 250$ (approximately 2 US\$) a cotton.

Considering TC is just a common cotton stick, the surgeon can sense that he able to apply a delicate tension from any angle, allowing pinpoint exposure of the cutting line. If gauze or sponge is used to rotate the pancreas, it is necessary to grasp it over and over again. We realize how comfortable the uninterrupted view is with less stress. Even then with a swollen pancreas, there is no need to crazily scramble to compress the pancreas and get anxious about the damage to the pancreas, because the root of TC can hook on the edge of the pancreas and rotate it regionally. Also, residents who are not used to manipulate the laparoscopic forceps are able to secure a stable surgical field.

Furthermore, our results showed that there was only one case of Grade 2 pancreatic fistula in 34 cases, and we seemed to be able to minimize the frequency and severity of postoperative pancreasrelated complications. Previous reports including large clinical trials indicate that frequency of postoperative pancreas-related complications is varied (1.0-8.4\%), and little difference exists between open surgery and laparoscopic surgery [10-12] but it is hard to ignore a small but significant complication. Especially, some authors have reported that LG significantly increase the level of d-AMY than open gastrectomy (OG)[10]. We emphasize that it is important to improve surgical safety and even a little preventive measures of postoperative pancreas-related complications. Recently, Tsujiura et al. presented a new method by pulling and controlling the connective tissues along the inferior border of the pancreas to prevent postoperative infectious complications [13]. They showed that pancreas compression had a huge effect on postoperative infectious complications.

Other factors affecting postoperative pancreas-related complications can be regarded as surgeon's technical learning curve, lateral thermal damage to the pancreas by various energy devices, the depth of lymphadenectomy, and anatomical characteristics of the pancreas [14-16]. We were able to achieve a good outcome, because the delicate compression with TC could minimize the pancreatic injury and be provided a good field of view easily. Since we need to obtain further traction and tension in deep D2 lymphadenectomy, we sometimes switch the TC to grasping forceps in order to grasp and pull the periarterial nerves along the common hepatic and splenic arteries. While bearing in mind the unsuitableness for deep lymphadenectomy, we try to select the method on a case-by-case basis.

\section{Conclusion}

TC should be considered as a useful option in the safe and delicate pancreatic rotating technique for suprapancreatic lymphnode dissection in LG.

\section{Competing Interests}

The authors have declared that no competing interest exists.

\section{References}

1. Yamaguchi T, Fukunaga T, Hiki N (2011) Laparoscopic Gastric Surgical Procedures: Distinctive (Innovative) Operative Style of Cancer Institute Hospital, Medical View, Japan, pp. 56-69.

2. Eto T, Kitano S (2011) Laparoscope assisted distal gastrectomy. Gastroenterological Surgery 34:752-760.

3. Nobuoka T, Ito T, Kyuno T, Nishidate T, Imamura M, et al. (2014) Laparoscopic distal gastrectomy. Gastroenterological Surgery 37: 17751783.

4. Shimada M, Amaya S, Sugita H, Furutani Y, Suzuki H, et al. (2015) Safe rotation technique with Cat Hand for suprapancreatic lymphnode dissection in laparoscopic gastrectomy. Hokuriku Geka Gakkai 34:7-9.

5. Japanese Gastric Cancer Association. (2010) Japanese Classification of Gastric Carcinoma.14th ed. Kanehara Syuppan, Japan, pp.1-26.

6. Bassi C, Dervenis C, Butturini G, Fingerhut A, Yeo C, et al. (2005) Postoperative pancreatic fistula: an international study group (ISGPF) definition. Surgery 138: 8-13. 
Citation: Shimada M, Amaya S, Tatemichi K, Tawara H, Furutani Y, et al. (2017) Delicate Pancreatic Rotating Technique Using "Thoraco Cotton" in Laparoscopic Gastrectomy. Int J Gastroenterol Disord Ther 4: 133. doi: https://doi.org/10.15344/2393-8498/2017/133

Page 6 of 6

7. Dindo D, Demartines N, Clavien PA. (2004) Classification of surgical complications: a new proposal with evaluation in a cohort of 6336 patients and results of a survey. Ann Surg 240: 205-213.

8. Japanese Gastric Cancer Association. (2014) Gastric Cancer Treatment Guidelines 4th ed. Tokyo: Kanehara Syuppan, Japan; pp.17-18

9. Japan Society for Endoscopic Surgery (2014) Endoscopic Surgery Guidelines. Tokyo: Wiley Publishing Japan, Japan, pp.12-17.

10. Obama K, Okabe H, Hosogi H, Tanaka E, Itami A, et al. (2011) Feasibility of laparoscopic gastrectomy with radical lymph node dissection for gastric cancer: from a viewpoint of pancreas-related complications. Surgery 149:15-21.

11. Katai H, Sasako M, Fukuda H, Nakamura K, Hiki N, et al. (2010) Safety and feasibility of laparoscopy-assisted distal gastrectomy with suprapancreatic node dissection for clinical stage I gastric cancer: a multi center phase I trial (JCOG 0703). Gastric Cancer 13: 238-244.

12. Takemura M, Toyokawa T, Sakurai K, Yoshida K, Takii M, et al. (2010) Clinical Outcomes after Laparoscopic Distal Gastrectomy for pT1 or pT2 Gastric Cancer. Jpn J Cancer Clin 56: 849-856.

13. Tsujiura M, Hiki N, Ohashi M, Nunobe S, Kumagai K, et al. (2017) "PancreasCompressionless Gastrectomy": A Novel Laparoscopic Approach for Suprapancreatic Lymph Node Dissection.. Ann Surg Oncol 24: 3331-3337.

14. Gao T, Lau BE, Yamaguchi T, Hanari N, Gunji H, et al. (2016) Experimenta analyses of the cavitation generated by ultrasonically activated surgical devices. Surg Today 47: 122-129.

15. Migita K, Matsumoto S, Wakatsuki K, Ito M, Kunishige T, et al. (2016) The anatomical location of the pancreas is associated with the incidence of pancreatic fistula after laparoscopic gastrectomy. Surg Endosc 30: 54815489.

16. Kobayashi N, Shinohara H, Haruta S, Ohkura Y, Mizuno A, et al. (2016) Process of pancreas head as a risk factor for postoperative pancreatic fistula in laparoscopic gastric cancer surgery. World J Surg 40: 2194-201. 